\title{
NIPPLE-SPARING MASTECTOMY WITH IMMEDIATE IMPLANT-BASED RECONSTRUCTION FOR PATIENTS WITH PURE DUCTAL CARCINOMA IN SITU
}

Antônio Luiz Frasson'1, Ana Beatriz Falcone², Isabela Miranda³ , Betina Vollbrecht Mônica Adriana Rodriguez Martinez Frasson ${ }^{1}$

${ }^{1}$ Hospital Albert Einstein - Porto Alegre (RS), Brazil.

${ }^{2}$ Hospital Albert Einstein - São Paulo (SP), Brazil.

${ }^{3}$ Pontifícia Universidade Católica - Porto Alegre (RS), Brazil.

Introduction: The presence of extensive intraductal component is strongly associated to the increasing risk of nippleareola complex (NAC) relapse. Most of the studies focusing on the outcomes of patients diagnosed with pure ductal carcinoma in situ (DCIS) who underwent nipple-sparing mastectomy (NSM) available in the literature performed immediate breast reconstruction using prosthetic implants (saline-filled implant or tissue expander) and autologous tissue flap. Objectives: The aim of our study was to evaluate the outcomes of patients diagnosed with DCIS who underwent NSM with immediate implant-based breast reconstruction. Methods: We retrospectively analyzed complication rates and oncological safety of 67 breast cancer patients diagnosed with pure DCIS who underwent NSM with immediate implantbased breast reconstruction between 2004 and 2018. Patients who underwent risk reduction NSM with accidental finding of DCIS were included in the study, and all patients were operated by the lead author. Tumor-nipple distance and tumor size were not exclusion criteria. Results: The indications of NSM were risk reduction breast surgery with accidental finding of DCIS ( $n=4.6 \%)$, multifocal disease $(n=16,23.9 \%)$, compromised margins after breast-conserving surgery $(n=11,16.4 \%)$, tumors $>40 \mathrm{~mm}(\mathrm{n}=16,23.9 \%)$ and unfavorable relationships between tumor size and breast size or patient preference $(n=20,29.8 \%)$. The mean age of the patients was 46.8 years (range 30-75). Of the 69 procedures performed for DCIS, a total of three complications occurred, including two hematomas (2.9\%) and one partial nipple necrosis (1.4\%). After a mean follow-up of 60 months (range 3-183 months) the local recurrence rate was $8.9 \%$. We observed a tendency of tumor multifocality being a risk factor for local relapse. The disease-free survival (DFS) rate was $90 \%$ and none of the patients died during the follow-up. Conclusions: Despite the local recurrence rate, we showed that NSM with immediate breast reconstruction using prosthetic implant is a feasible surgical approach with low complication rates and good disease-free and overall survival rates for patients with pure DCIS when BCS is not an option. 